\title{
THE COMPACTNESS OF THE SET OF ARC CLUSTER SETS OF AN ARBITRARY FUNCTION
}

\author{
JOHN T. GRESSER
}

\begin{abstract}
It is known that if $f$ is a continuous complex-valued function defined in the open unit disk $D$, then the set $\mathcal{C}_{f}(\zeta)(\zeta \in \partial D)$ of all arc cluster sets of $f$ at $\zeta$ is compact in a natural topology for all but at most a countable number of points $\zeta \in \partial D$. We show that if $f$ is an arbitrary complex-valued function defined on an arbitrary subset $Z$ of the plane, then $\mathfrak{C}_{f}(p)$ is compact for all but at most a countable number of points $p \in Z \cup \partial Z$.
\end{abstract}

1. Introduction. Let $\mathfrak{S}$ be the collection of all closed, nonempty subsets of the Riemann sphere $W$. We define what is known as the Hausdorff metric on $\mathfrak{S}$ by

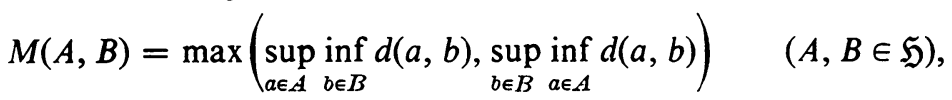

where $d(a, b)$ is the spherical distance between $a$ and $b$. With this metric $\mathfrak{S}$ is a compact metric space.

Now let $P$ denote the complex plane, and let $p \in P$. We say that $\alpha$ is an arc at $p$ if $\alpha \subseteq P-\{p\}$ and is the image of a continuous function $z=z(t)$ $(0 \leqq t<1)$ such that $z(t) \rightarrow p$ as $t \rightarrow 1$. We call $\alpha$ a simple arc at $p$ if $\alpha$ is in addition homeomorphic to $[0,1)$. If $Z$ is any nonempty subset of $P$, we let $Z$ denote the closure of $Z$ and define, for $p \in Z$,

$$
\mathscr{A}_{p}(Z)=\{\alpha: \alpha \text { is a simple arc at } p \text { with } \alpha \subseteq Z\} \text {. }
$$

We assume that the reader is familiar with the elementary notions of cluster set theory (see [3] or [4]). If $f$ is an arbitrary function whose domain is $Z$ and whose range is a subset of $W$, if $p \in Z$ and $\alpha \in \mathscr{A}_{p}(Z)$, then $C(f, p, \alpha)$ denotes the arc cluster set of $f$ at $p$ along $\alpha$. We let $\mathbb{C}_{f}(p)=$ $\left\{C(f, p, \alpha): \alpha \in \mathscr{A}_{p}(Z)\right\}$. Then $\mathfrak{H}$ topologizes the set $\mathfrak{C}_{f}(p)$ with what has been called the $M$-topology.

This paper is written in response to the following two theorems which appear in [2, Theorems 1 and 2, pp. 211, 213].

Received by the editors November 12, 1971 and, in revised form, April 17, 1972. AMS 1970 subject classifications. Primary 30A72; Secondary 30A90.

Key words and phrases. Arc cluster set, set of arc cluster sets of an arbitrary function, selector of arcs, missing arc cluster set.

(c) American Mathematical Society 1973 
THEOREM A. Let $f$ be a continuous function in $D$, and let $\zeta \in K$. If $\zeta$ is not an ambiguous point of $f$, then $\mathfrak{C}_{f}(\zeta)$ is a compact set in the $M$-topology.

THEOREM B. There is a function $f$ defined in $D$ such that $\zeta_{0}=1$ is not an ambiguous point of $f$, and $\mathbb{C}_{f}(1)$ is not a compact set in the $M$-topology.

Since an arbitrary function in $D$ can have at most a countable number of ambiguous points $\zeta \in K$ [1, Theorem 2, p. 380], it follows from Theorem A that if $f$ is a continuous function in $D$, then $\mathfrak{C}_{f}(\zeta)$ is a compact set in the $M$-topology for all but at most a countable number of points $\zeta \in K$. This raises the question as to whether or not $\mathfrak{C}_{f}(\zeta)$ is a compact set in the $M$ topology for all but at most a countable number of points $\zeta \in K$ when $f$ is an arbitrary complex valued function defined in $D$. But since the boundary of a domain plays no preferential role insofar as an arbitrary function is concerned, it is natural to investigate the question of compactness of $\mathfrak{C}_{f}(p)$ for points $p$ interior to $D$ as well. Although the result in this paper deals with an arbitrary domain, our most striking conclusion is that if $f$ is an arbitrary function defined in the plane $P$, then $\mathfrak{C}_{f}(p)$ is a compact set in the $M$-topology for all but at most a countable number of points $p \in P$. Our result, in addition, answers the above question concerning the unit circle $K$ in the affirmative.

2. Geometry of arcs. Our proof requires several rather specialized definitions. If $\alpha$ is an arc at $p \in P$, we say that $\beta$ is a terminal subarc of $\alpha$ if $\beta=z\left(\left[t_{0}, 1\right)\right)$ for some $t_{0}$ and some representative $z=z(t)$ of $\alpha$. We call $\gamma$ a curvilinear segment if $\gamma$ is the image of a continuous function $z=z(t)$ $(0 \leqq t \leqq 1)$. A sequence $\left\langle\gamma_{j}\right\rangle$ of curvilinear segments is said to converge to $p \in P$ provided that $p \notin \gamma_{j}$ for every $j$, and that for every $\varepsilon>0$ all but a finite number of the sets $\gamma_{j}$ are contained in the open disk of radius $\varepsilon$ centered at $p$.

Let $E \subseteq P$. We say that $\Gamma$ is a selector of arcs on $E$ if for every $p \in E$, $\Gamma(p)$ is a nonempty collection of simple arcs at $p$, and if $\Gamma(p)=\varnothing$ for every $p \notin E$. If $Q \subseteq P$ and $\alpha, \beta$ are arcs at $p$, we say that $Q$ joins $\alpha$ and $\beta$ if there is a sequence $\left\langle\gamma_{j}\right\rangle$ of curvilinear segments such that $\gamma_{j} \subseteq Q$ for every $j$, $\left\langle\gamma_{j}\right\rangle$ converges to $p$, and $\gamma_{j} \cap \alpha \neq \varnothing \neq \gamma_{j} \cap \beta$ for every $j$. If $\Gamma$ is a selector of arcs on $E, Q \subseteq P$, and $p \in E$, we say that $Q$ joins $\Gamma(p)$ if $Q$ joins $\alpha$ and $\beta$ for every $\alpha, \beta \in \Gamma(p)$. If $\Gamma$ is a selector of arcs on $E$, we define the sets

$$
\Sigma \Gamma(p)=\bigcup_{\alpha} \alpha \quad(\alpha \in \Gamma(p)), \quad \Sigma \Gamma=\bigcup_{p} \Sigma \Gamma(p) \quad(p \in E) .
$$

LEMMA 1. Let $E \subseteq P$ and let $\Gamma$ be a selector of arcs on $E$ such that the cardinality of $\Gamma(p)$ is six for every $p \in E$. Suppose there is an $\varepsilon>0$ such that $\operatorname{dia}(\alpha) \geqq \varepsilon$ for every $\alpha \in \Gamma(p)$, and every $p \in E$. Then there is a set $F \subseteq E$, with $E-F$ at most countable, such that for every $p \in F$ there are distinct 
arcs $\alpha, \beta \in \Gamma(p)$, and there is a curvilinear segment $\gamma \subseteq \Sigma \Gamma$ satisfying $p \notin \gamma$, $\operatorname{dia}(\gamma) \leqq \varepsilon$, and $\gamma \cap \alpha \neq \varnothing \neq \gamma \cap \beta$.

Proof. If the lemma is false, then there is an uncountable subset $G$ of $E$ such that for every $p \in G$ and every pair $\alpha, \beta$ of distinct arcs in $\Gamma(p)$ no such curvilinear segment exists. By covering $G$ with a countable collection of open disks of diameters $\varepsilon$, it follows that for at least one such disk, say $D_{0}$, the set $G_{0}=G \cap D_{0}$ is uncountable. For each $p \in G_{0}$, and each $\alpha \in$ $\Gamma(p), \alpha$ intersects the boundary $C_{0}$ of $D_{0}$, so that there is a terminal subarc of $\alpha$ which is contained in $D_{0}$ except for one end point which lies on $C_{0}$. Let $\alpha_{j}(p)(j=1,2, \cdots, 6)$ denote these terminal subarcs of arcs in $\Gamma(p)$ for $p \in G_{0}$.

Now let $\left\{A_{j}\right\}$ be a countable collection of open circular subarcs of $C_{0}$ which forms a basis for the usual topology on $C_{0}$. For each $p \in G_{0}$, by choosing the smallest indices possible, let $A_{i}(p)(i=1,2, \cdots, 6)$ be mutually disjoint members of $\left\{A_{j}\right\}$ such that $\alpha_{i}(p)$ intersects $A_{i}(p)$. Since the collection of all 6-tuples having coordinates in $\left\{A_{j}\right\}$ is countable, it follows that we can find distinct points $p, q \in G_{0}$ such that $A_{i}(p)=A_{i}(q)$ for each $i=1,2, \cdots, 6$. We assert that this is impossible.

A trivial consequence of a point $r$ being in $G$ is that no two arcs in $\Gamma(p)$ intersect. Consequently the set $\left(D_{0}-\{p\}\right)-\bigcup_{j=1}^{6} \alpha_{j}(p)$ contains six components, say $D_{j}(j=1,2, \cdots, 6)$, each of which is bounded by two of the arcs $\alpha_{k}(p)(k=1,2, \cdots, 6),\{p\}$, and a circular subarc of $C_{0}$.

There are two cases to consider. If $q$ lies in one of the domains, say $D_{j_{0}}$, then at least four of the arcs $\alpha_{k}(q)$ must cross the boundary of $D_{j_{0}}$. Since these arcs are mutually disjoint, only one can cross the boundary of $D_{j_{0}}$ at $p$. Therefore at least two of the $\operatorname{arcs} \alpha_{k}(q)$ must cross one of the $\operatorname{arcs} \alpha_{j}(p)$, and this contradicts $q \in G$. In the other case, $q$ lies on one of the arcs at $p$, say $\alpha_{j_{0}}(p)$. We let $D^{\prime}$ be the union of $\alpha_{j_{0}}(p)$ with its two adjacent domains. Now at least two of the arcs $\alpha_{k}(q)$ must cross the boundary of $D^{\prime}$ at points distinct from $p$. No two of the arcs $\alpha_{k}(q)$ can cross one of the $\operatorname{arcs} \alpha_{j}(p)$ since $q \in G$. Hence two of the arcs $\alpha_{k}(q)$ cross two of the arcs $\alpha_{j}(p)$. But since $q \in \Sigma \Gamma$ this implies that $q$, together with two of the arcs $\alpha_{k}(q)$, form a curvilinear segment which contradicts $p \in G$, and this completes the proof.

REMARK. It can be shown that Lemma 1 is false if the cardinality condition is replaced by a cardinality of four. On the other hand, it can be shown that Lemma 1 remains true if the cardinality condition is replaced by a cardinality of five. The merits of this stronger result, however, do not justify the added difficulties encountered in the proof.

Lemma 2. Let $E \subseteq P$ and let $\Gamma$ be a selector of arcs on $E$ such that the cardinality of $\Gamma(p)$ is six for every $p \in E$. Then there is a set $F \subseteq E$, with 
$E-F$ at most countable, such that for every $p \in F$ there are distinct arcs $\alpha$ and $\beta$ in $\Gamma(p)$ such that $\Sigma \Gamma$ joins $\alpha$ and $\beta$.

Proof. For each $j=1,2,3, \cdots$, let

$$
E_{j}=\{p \in E: \operatorname{dia}(\alpha) \geqq 1 / j \text { for every } \alpha \in \Gamma(p)\},
$$

so that $E$ is the union of the sets $E_{j}$. According to Lemma 1, for each $j=1,2,3, \cdots$, and each $k \geqq j$ there is a set $F_{j}(k) \subseteq E_{j}$, with $E_{j}-F_{j}(k)$ at most countable, and for every $p \in F_{j}(k)$ there is a curvilinear segment $\gamma \subseteq \Sigma \Gamma$, and distinct arcs $\alpha, \beta \in \Gamma(p)$ such that $p \notin \gamma, \operatorname{dia}(\gamma) \leqq 1 / k$, and $\alpha \cap \gamma \neq \varnothing \neq \beta \cap \gamma$. We set

$$
F=\bigcup_{j=1}^{\infty} \bigcap_{k=j}^{\infty} F_{j}(k),
$$

so that $E-F$ is at most countable. Since $\Gamma(p)$ is a finite set, it now follows that $\Sigma \Gamma$ joins at least two distinct arcs in $\Gamma(p)$ for every $p \in F$.

LEMMA 3. Let $E \subseteq P$ and let $\Gamma$ be a selector of arcs on $E$. Then there is $a$ set $F \subseteq E$, with $E-F$ at most countable, and there are selectors of arcs $\Gamma_{j}$ $(j=1,2, \cdots, 5)$ on $E$ such that $\Gamma(p)=\bigcup_{j=1}^{5} \Gamma_{j}(p)$ for every $p \in E$, and $\Sigma \Gamma$ joins $\Gamma_{j}(p)$ for every $p \in F$ and $j=1,2, \cdots, 5$.

Proof. Let $p \in E$, and let $\alpha, \beta \in \Gamma(p)$. Define $\alpha \sim \beta$ if and only if $\Sigma \Gamma$ joins $\alpha$ and $\beta$. This defines an equivalence relation on $\Gamma(p)$. Let $F$ be the set of all points $p \in E$ for which $\Gamma(p)$ contains less than six equivalence classes. For each $p \in E-F$ let $\alpha_{j}(p)(j=1,2, \cdots, 6)$ be members of six different equivalence classes of $\Gamma(p)$, and define

$$
\Gamma_{0}(p)=\left\{\alpha_{j}(p): j=1,2, \cdots, 6\right\} \quad(p \in E-F) .
$$

Then $\Gamma_{0}$ is a selector of arcs on $E-F$ such that $\Sigma \Gamma_{0}$ joins no two arcs in $\Gamma_{0}(p)$ for every $p \in E-F$. It follows from Lemma 2 that $E-F$ is at most countable. For each $p \in E-F$ define $\Gamma_{j}(p)=\Gamma(p)(j=1,2, \cdots, 5)$. For each $p \in F$ let $\Gamma_{j}(p) \quad(j=1,2, \cdots, 5)$ denote the various equivalence classes in $\Gamma(p)$, where duplication is allowed in the case that $\Gamma(p)$ has less than five equivalence classes. It follows that $\Sigma \Gamma$ joins $\Gamma_{j}(p)$ for every $p \in F$ and every $j=1,2, \cdots, 5$.

3. The main result. Let $Z$ be an arbitrary subset of $P$, and let $f$ be an arbitrary complex valued function defined on $Z$. We call a closed subset $C$ of the Riemann sphere $W$ a missing arc cluster set of $f$ at $p \in Z$ if $C \in$ $\mathbb{\mathfrak { C }}_{f}(p)-\mathfrak{C}_{f}(p)$, where $\overline{\mathfrak{C}}_{f}(p)$ denotes the closure of $\mathfrak{C}_{f}(p)$ in $\mathfrak{H}$. If $A \subseteq Z$ we let $\bar{f}(A)$ denote the closure of $f(A)$ in $W$. If $C$ is a closed, nonempty subset of $W$, and $\varepsilon>0, C(\varepsilon)$ will denote the set of all points whose spherical distance from $C$ does not exceed $\varepsilon$. It follows that if $P, Q \in \mathfrak{H}$, then $M(P, Q) \leqq \varepsilon$ if and only if $P \subseteq Q(\varepsilon)$ and $Q \subseteq P(\varepsilon)$. Several immediate 
properties of $M$ follow from this observation, and we will use them freely and without reference in the next proof.

THEOREM 1. Let $f$ be an arbitrary complex valued function defined on an arbitrary subset $Z$ of the plane. Then $\mathfrak{C}_{f}(p)$ is a compact set in the $M$ topology for all but at most a countable number of points $p \in Z$.

Proof. Let $E$ be the set of all points in $Z$ at which $\mathfrak{C}_{f}(p)$ is not compact, and for each $p \in E$, let $C_{p}$ be a missing arc cluster set of $f$ at $p$. Let $\left\langle\varepsilon_{j}\right\rangle$ be a decreasing sequence of positive numbers which converges to zero. Then for each $j=1,2,3, \cdots$, and each $p \in E$, let $\alpha_{j}(p) \in \mathscr{A}_{p}(Z)$ such that

$$
\begin{gathered}
M\left(C\left(f, p, \alpha_{j}(p)\right), C_{p}\right) \leqq \varepsilon_{j} / 4, \\
M\left(\bar{f}\left(\alpha_{j}(p)\right), C_{p}\right) \leqq \varepsilon_{j} / 2 .
\end{gathered}
$$

For each $k=1,2,3, \cdots$ let $\mathfrak{5}(k, 1), \mathfrak{G}(k, 2), \cdots, \mathfrak{G}\left(k, n_{k}\right)$ be a covering of $\mathfrak{H}$ by compact neighborhoods such that

$$
\operatorname{dia}(\mathfrak{G}(k, n)) \leqq \varepsilon_{k} / 2 \quad\left(n \leqq n_{k}\right) .
$$

Then define

(4) $L(k, n)=\left\{p \in E: C_{p} \in \mathbb{F}(k, n)\right\} \quad\left(k=1,2,3, \cdots ; n \leqq n_{k}\right)$.

We let $\Gamma[k, n]$ be the selector of arcs on $L(k, n)$ defined by

(5) $\Gamma[k, n](p)=\left\{\alpha_{j}(p): j=k, k+1, k+2, \cdots\right\} \quad(p \in L(k, n))$.

It follows from (2)-(5) and the properties of $M$ that

$$
\bar{f}(\Sigma \Gamma[k, n]) \subseteq C_{p}\left(\varepsilon_{k}\right) \quad\left(p \in L(k, n), n \leqq n_{k}\right) .
$$

According to Lemma 3 , for each $k=1,2,3, \cdots$, and $n \leqq n_{k}$, there is a set $F(k, n) \subseteq L(k, n)$, with $L(k, n)-F(k, n)$ at most countable, and selectors of arcs $\Gamma_{j}[k, n](j=1,2, \cdots, 5)$ on $L(k, n)$ such that

and

$$
\Gamma[k, n](p)=\bigcup_{j=1}^{5} \Gamma_{j}[k, n](p) \quad(p \in L(k, n)),
$$

(8) $\Sigma \Gamma[k, n]$ joins $\Gamma_{j}[k, n](p) \quad(p \in F(k, n), j=1,2, \ldots, 5)$.

Define the set $F_{k}=\bigcup_{n=1}^{n_{k}} F(k, n)$, and note that each of the sets $F_{k}$ contains all but at most a countable number of points in $E$ since, for each $k$, $E=\bigcup_{n=1}^{n_{k}} L(k, n)$. Therefore the set $F=\bigcap_{k=1}^{\infty} F_{k}$ contains all but at most a countable number of points in $E$.

We will show that $E$ is countable by showing that $F$ is empty. Suppose to the contrary that $p \in F$. We assert that there is an $\alpha \in \mathscr{A}_{p}(Z)$ whose corresponding arc cluster set is $C_{p}$. 
Since $p \in F$ it follows that for every $k=1,2,3, \cdots$, there is an $m_{k} \leqq n_{k}$ such that $p \in F\left(k, m_{k}\right)$. Using (8) this means that, for every $k=1,2,3, \cdots$, $\Sigma \Gamma\left[k, m_{k}\right]$ joins $\Gamma_{j}\left[k, m_{k}\right](p)(j=1,2, \cdots, 5)$. It follows from (5) and (7) that if $j$ is an integer for which $\Gamma_{j}\left[k, m_{k}\right](p)$ is an infinite set, then there is an integer $i$ such that

$$
\Gamma_{j}\left[k, m_{k}\right](p) \cap \Gamma_{i}\left[k+1, m_{k+1}\right](p)
$$

is an infinite set. Using this observation, we construct inductively sets $\Lambda_{k}=\Gamma_{j_{k}}\left[k, m_{k}\right](p)(k=1,2,3, \cdots)$ in such a way that $\Lambda_{k} \cap \Lambda_{k+1}$ is an infinite set for each $k$. We then define inductively a sequence $\left\langle\alpha_{k}\right\rangle$ of arcs $\alpha_{k} \in \Lambda_{k} \cap \Lambda_{k+1}$ in such a way that $\left\langle\alpha_{k}\right\rangle$ is a subsequence of $\left\langle\alpha_{k}(p)\right\rangle$. From our construction it follows that $\Sigma \Gamma\left[k, m_{k}\right]$ joins $\alpha_{k-1}$ and $\alpha_{k}(k=2,3$, $4, \cdots)$. For each $k=2,3,4, \cdots$, let $\left\langle\gamma_{j}(k)\right\rangle$ be a sequence of curvilinear segments such that

$$
\begin{aligned}
\gamma_{j}(k) \subseteq \Sigma \Gamma\left[k, \dot{m}_{k}\right] . \quad(j=1,2,3, \cdots), \\
\left\langle\gamma_{j}(k)\right\rangle \text { converges to } p \quad(k \text { fixed }), \\
\gamma_{j}(k) \cap \alpha_{k-1} \neq \varnothing \neq \gamma_{j}(k) \cap \alpha_{k} \quad(j=1,2,3, \cdots) .
\end{aligned}
$$

It follows from (6) and (9) that

$$
\bar{f}\left(\gamma_{j}(k)\right) \subseteq C_{p}\left(\varepsilon_{k}\right) \quad(j, k=1,2,3, \cdots) .
$$

Then using (1), (10), (11), (12), and the definition of an arc cluster set, a simple arc $\alpha$ at $p$ can be constructed which passes through the sets $\alpha_{k}$ and the sets $\gamma_{j}(k)$, and satisfies $C(f, p, \alpha)=C_{p}$. This contradicts the definition of $C_{p}$ and completes the proof.

\section{REFERENCES}

1. F. Bagemihl, Curvilinear cluster sets of arbitrary functions, Proc. Nat. Acad. Sci. U.S.A. 41 (1955), 379-382. MR 16, 1095.

2. C. Belna and P. Lappan, The compactness of the set of arc cluster sets, Michigan Math. J. 16 (1969), 211-214. MR 39 \#7111.

3. E. F. Collingwood and A. J. Lohwater, The theory of cluster sets, Cambridge Tracts in Math. and Math. Phys., no. 56, Cambridge Univ. Press, Cambridge, 1966. MR 38 \#325.

4. K. Noshiro, Cluster sets, Ergebnisse der Mathematik und ihrer Grenzgebiete, N.F., Heft 28, Springer-Verlag, Berlin, 1960. MR 24 \#A3295.

Department of Mathematics, Bowling Green State University, Bowling Green, OHIO 43403 